\title{
Uso de la endoscopia por síntomas de reflujo gastroesofágico en una muestra de población adulta mexicana
}

\author{
Raúl Contreras-Omaña ${ }^{1 *}$, Isis Silva-de la Vega ${ }^{1,2}$, José A. Velarde-Ruíz Velasco ${ }^{3}$, \\ Diana Madrigal-Sánchez ${ }^{4}$, Ma. Fatima Higuera-de la Tijera ${ }^{5}$, Felipe Zamarripa-Dorsey ${ }^{6}$, \\ Graciela E. Castro-Narro7, Stephany Téllez-Jaén ${ }^{1}$ y Juan F: Martínez-Campos ${ }^{2}$
}

${ }^{1}$ Servicio de Hepatología, Centro de Investigación en Enfermedades Hepáticas y Toxicológicas, Pachuca de Soto, Hgo.; ${ }^{2}$ Área Académica de Medicina, Instituto de Ciencias de la Salud, Universidad Autónoma del Estado de Hidalgo, Pachuca de Soto, Hgo.; ${ }^{3}$ Servicio de Gastroenterología, Hospital Civil Fray Antonio Alcalde, Guadalajara, Jal.; ${ }^{4}$ Servicio de Urgencias, Hospital General de Zona y Medicina Familiar N. ${ }^{\circ} 8$, Instituto Mexicano del Seguro Social, Ciudad de México; ${ }^{5}$ Servicio de Gastroenterología, Hospital General de México, Ciudad de México; ${ }^{6}$ Servicio de Gastroenterología, Hospital Juárez de México, Ciudad de México; ${ }^{7}$ Servicio de Gastroenterología, Instituto Nacional de Ciencias Médicas y Nutrición Salvador Zubirán, Ciudad de México. México

\section{Resumen}

Objetivo: Conocer el uso que se hace de la endoscopia por síntomas de reflujo gastroesofágico en una muestra de población adulta mexicana. Método: Estudio prospectivo, observacional, transversal y descriptivo. Se aplicó una encuesta a 1,502 personas de ambos sexos mayores de 18 años en el periodo julio del 2018 a mayo del 2019 en México. Se extendió por medio de redes sociales y se repartió afuera de escuelas, consultorios y centros de salud. Resultados: Se aplicaron 1,502 encuestas. Del total, el $61 \%$ fueron del sexo femenino. Doscientos treinta individuos (37.70\%) se han realizado al menos una endoscopia por síntomas de reflujo en los últimos cinco años, comparado con más del $60 \%$ reportado en la literatura internacional. De los pacientes con enfermedad por reflujo gastroesofágico a 136 se les ha realizado endoscopia en una ocasión y a 94 en dos ocasiones o más. De entre los estudios para diagnosticar enfermedad por reflujo, la endoscopia es el más utilizado, representando el $52.4 \%$ de total de estudios solicitados a estos pacientes. En los 230 pacientes a quienes se realizó endoscopia se llevaron a cabo durante los últimos cinco años un total de 365 procedimientos (1.58 endoscopias por paciente), número considerablemente mayor a lo reportado en la literatura internacional (1.04 endoscopias por paciente, $p=0.05$ ). Conclusiones: $E I$ uso de la endoscopia como método diagnóstico inicial en pacientes con síntomas de reflujo en México parece ser menor a lo reportado en la literatura internacional, aunque el número total de procedimientos por paciente es significativamente mayor.

Palabras clave: Endoscopia. Reflujo gastroesofágico. México.

\section{Endoscopy use for gastroesophageal reflux symptoms in a Mexican adult population sample}

\section{Abstract}

Objective: To determine the use of endoscopy in a Mexican adult population sample due to gastroesophageal reflux symptoms. Methods: A prospective, observational, transversal and descriptive study where a survey was applied to 1502 people

Disponible en internet: 12-03-2021

Endoscopia. 2021;33(1):45-49 www.endoscopia-ameg.com 
of both sexes and over 18 years in the period July 2018-May 2019 in Mexico. It was extended through social networks and distributed outside schools, clinics and healthcare centers. Results: 1502 surveys were applied. From the whole sample $61 \%$ were female. 230 individuals (37.70\%) had an endoscopy due to reflux symptoms in the last five years compared to more than 60\% reported in the international literature. Of the patients with gastroesophageal reflux disease, 136 had an endoscopy on one occasion and 94 twice or more. The endoscopy was the most used diagnostic method among all, due to reflux symptoms representing $52.4 \%$ of the total. In the 230 patients who underwent to an endoscopy, a total of 365 procedures (1.58 endoscopies per patient) were realized during the last five years, a considerably larger number than reported in the international literature (1.04 endoscopies per patient, $p=0.05$ ). Conclusions: The use of endoscopy as an initial diagnostic method in patients with reflux symptoms in Mexico seems to be lower than that reported in the international literature, although the total number of procedures per patient is significantly higher.

Key words: Endoscopy. Gastroesophageal reflux. Mexico.

\section{Introducción}

La enfermedad por reflujo gastroesofágico (ERGE) se define como el ascenso del contenido gástrico o gastroduodenal por arriba de la unión gastroesofágica, que causa síntomas y/o daño estructural que afectan al bienestar y la calidad de vida de los individuos que la padecen ${ }^{1,2}$.

La endoscopia gastrointestinal ha tenido una consolidación manifiesta en el ámbito de la medicina nacional e internacional ${ }^{3}$. Su desarrollo, evolución y alcances han rebasado fronteras ${ }^{4}$. Por sí sola, posee baja sensibilidad en el diagnóstico de ERGE ${ }^{5}$, aunque es de elección para determinar la presencia de complicaciones como esofagitis, estenosis o esófago de Barrett. Sus hallazgos no tienen correlación con la intensidad de las manifestaciones clínicas que presenta el paciente. Está indicada cuando existen síntomas o signos de alarma y es recomendable realizarla si el paciente requerirá tratamiento prolongado 0 cuando este fracasa ${ }^{6-8}$.

Según las estadísticas del uso de la endoscopia en el mercado mexicano en los próximos años (20182024), la creciente carga de morbilidad junto con el rápido envejecimiento de la población será un factor clave que impulsará el crecimiento en el uso de dicho procedimiento en México ${ }^{9}$. De acuerdo con los datos de las Naciones Unidas sobre el envejecimiento de la población mundial, en México la población de 60 años y más representó 13.0 millones en 2017 y se estima que alcanzará los 40 millones en 2050. La población de ancianos propensos a sufrir enfermedades crónicas únicas o múltiples aumentará la demanda para procedimientos endoscópicos, lo que incentivará el crecimiento de la industria de la endoscopia en el país ${ }^{4,9}$. Sin embargo, hasta la fecha en México no hay estudios que reporten su uso actual por síntomas de reflujo gastroesofágico en la población general.

\section{Material y métodos}

Estudio prospectivo, observacional, transversal y descriptivo en el que se aplicó una encuesta autoevaluable a 1,502 personas de ambos sexos y de más de 18 años en el periodo de julio del 2018 a mayo del 2019 en México. La encuesta fue diseñada en Google For$\mathrm{ms}$, donde se incluyeron los cuestionarios Carlsson-Dent y GERD-Q (Gastroesophageal Reflux Disease Questionnaire) para conocer la prevalencia de ERGE en la muestra. A la población encuestada se le preguntó de manera directa lo siguiente: «¿Se le ha realizado alguna endoscopia por síntomas de reflujo como sensación de quemazón (agruras) detrás de su pecho; sensación de que se le regresa lo que comió o bebió a su garganta; dolor, ardor o molestia en la boca del estómago o náuseas?». De inicio se aplicó un piloto a un grupo pequeño de personas para validar las respuestas de la encuesta y posteriormente se extendió por medio de redes sociales ( $n=1,247,83 \%)$ y se repartió en escuelas, consultorios y centros de salud de Hidalgo y el Estado de México ( $n=255,17 \%)$. De las encuestas aplicadas en línea, respondieron individuos originarios de Ciudad de México ( $n=653,43.5 \%$ ), Jalisco $(n=222,14.8 \%)$, Sinaloa $(n=87,5.8 \%)$, Veracruz $(n=71,4.7 \%)$, Nuevo León $(n=63,4.2 \%)$, Yucatán $(n=51,3.4 \%)$, Querétaro $(n=48,3.2 \%)$, Baja California Norte $(n=37,2.5 \%)$ y Guerrero $(n=15,1 \%)$. No hubo diferencia significativa en edad y sexo de los encuestados entre las distintas regiones. Para el cálculo de la muestra para estudio observacional, se tomó como base el censo de población y vivienda del 2018 del Instituto Nacional de Estadística y Geografía, el cual reportó una población total en México de 123.5 millones de individuos, de los cuales se calcula que un $47 \%$ son mayores de 18 años, dando un total de 58 millones de personas. Basándonos en una prevalencia esperada de síntomas de reflujo a nivel poblacional de entre 


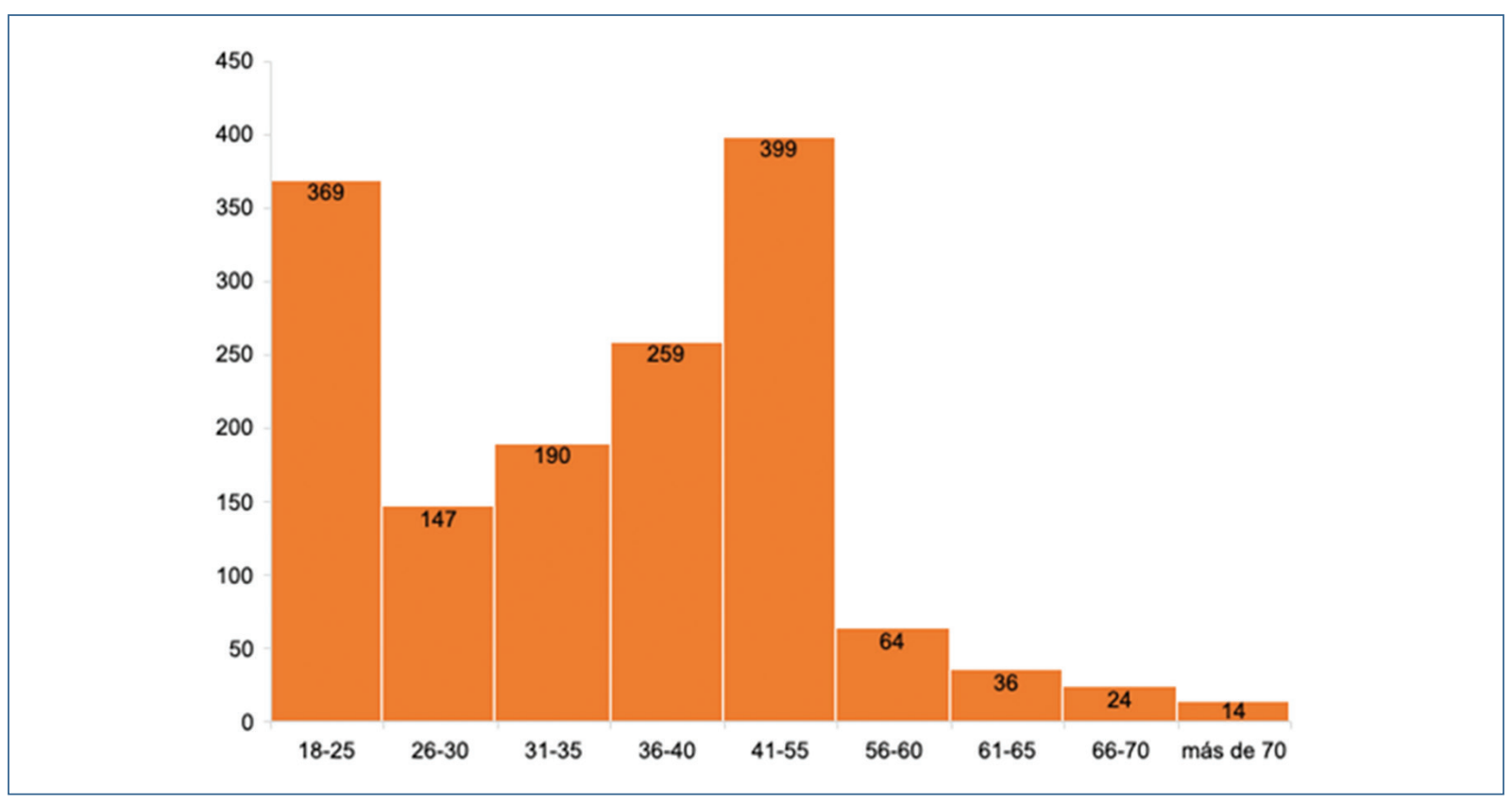

Figura 1. Edades de la muestra.

7 y $15 \%$ de acuerdo con lo indicado en el Consenso Mexicano de Enfermedad por Reflujo ${ }^{1}$, tomamos una media del 10\%; y con un riesgo de error aceptado del $5 \%$, la muestra que debíamos tomar para un nivel de confianza mayor al $99 \%$ era de 555 individuos. Los resultados se reportaron con medidas de tendencia central, dispersión y porcentajes.

\section{Resultados}

Se aplicaron 1,502 encuestas. Del total, el $61 \%$ fueron del sexo femenino y el $39 \%$ del masculino. El promedio de edad fue de 37.24 años, en donde el $26 \%$ tenía entre 41 y 55 años y el $25 \%$ entre 18 y 25 años (Fig. 1).

Doscientos treinta individuos (37.70\%) se han realizado al menos una endoscopia por síntomas de reflujo en los últimos cinco años. De los pacientes con ERGE, a $136(22.29 \%)$ se les ha realizado endoscopia en una ocasión y a $94(15.40 \%)$ en dos ocasiones o más. En la pregunta sobre los estudios que se han solicitado a los encuestados para diagnosticar enfermedad por reflujo, la endoscopia constituyó el $52.4 \%$ del total.

En los 230 pacientes a quienes se realizó endoscopia, se llevaron a cabo durante los últimos cinco años un total de 365 procedimientos (1.58 endoscopias por paciente) (Tabla 1, Fig. 2).

\section{Discusión}

Tomando en cuenta que la población de sexo femenino predomina en nuestro país $(51 \%)$ y siendo que los síntomas como pirosis tienden a presentarse con mayor frecuencia en el sexo femenino $0^{1,2}$ (a pesar de que las complicaciones de ERGE se presentan más en el sexo masculino), esperábamos mayor número de respuestas de mujeres en la encuesta, con un margen de $\pm 10 \%$.

De acuerdo con las recomendaciones de la Asociación Mexicana de Gastroenterología, la endoscopia no debe usarse de forma sistemática como prueba de escrutinio para ERGE por su pobre sensibilidad diagnóstica ${ }^{2,8,10}$. En nuestro estudio el $37.70 \%$ de la población encuestada con ERGE se ha realizado alguna endoscopia por síntomas de reflujo en los últimos cinco años y de este total a 136 se les ha realizado endoscopia en una ocasión y a 94 en dos ocasiones o más.

Según Schlottmann, et al., la endoscopia es frecuentemente la primera prueba realizada para confirmar la $E R G E^{11}$. $Y$ en nuestro trabajo de investigación fue el método de diagnóstico que más se utilizó entre todos los empleados por síntomas de reflujo, representando el $52.4 \%$ de total.

Un estudio en Dinamarca ${ }^{12}$ reportó que de un total de 36,292 pacientes, al 67.5\% se les realizó endoscopia 
Tabla 1. Estudios diagnósticos de enfermedad por reflujo gastroesofágico más solicitados los últimos cinco años

\begin{tabular}{|l|c|}
\hline Estudio & Porcentaje (\%) \\
\hline Esofagogastroduodenoscopia & 52.40 \\
\hline Serie esofagogastroduodenal & 13.06 \\
\hline Manometría esofágica & 9.61 \\
\hline pH-metría & 9.13 \\
\hline Otros & 15.8 \\
\hline Total & 100 \\
\hline
\end{tabular}

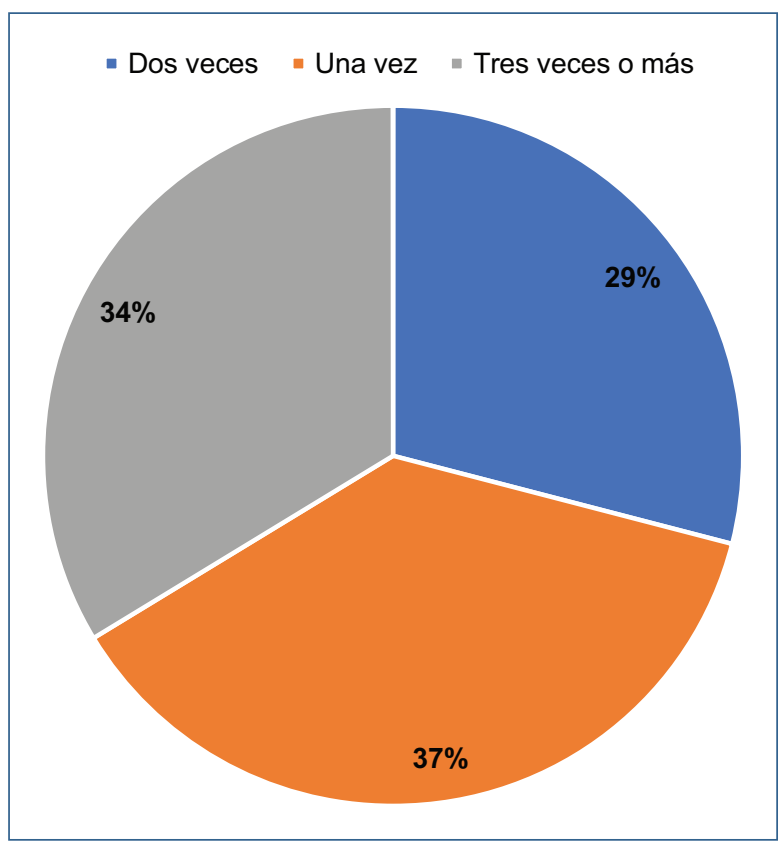

Figura 2. Endoscopias por síntomas de reflujo en los últimos cinco años.

sin biopsias de manera inicial para diagnosticar ERGE; considerablemente mayor al $37.70 \%$ reportado en nuestro estudio. Sin embargo, en la literatura internacional se reporta un número total de procedimientos por paciente significativamente menor (1.04 endoscopias por paciente, $p=0.05$ ) al descrito en nuestros resultados (1.58 endoscopias por paciente).

En aproximadamente el 50 al $60 \%$ de los pacientes con reflujo anormal, se ha evidenciado que no hay daño a la mucosa ${ }^{11,13}$. Por lo tanto, la Asociación Mexicana de Gastroenterología menciona una serie de indicaciones para la realización de la endoscopia digestiva alta, que son: ERGE de más de cinco años de evolución o
ERGE refractaria, en pacientes con signos de alarma como disfagia, hemorragia digestiva, dolor torácico y pérdida de peso no buscada, así como en aquellos pacientes con factores de riesgo para esófago de Barret y en los casos con sospecha de esofagitis eosinofílica². Nuestros resultados son importantes porque a pesar de estos datos, en nuestra muestra la endoscopia es el método de diagnóstico más utilizado en pacientes con síntomas de reflujo gastroesofágico, haciéndonos cuestionar si se tenían las indicaciones para su realización en todos los casos, o si en su lugar se trata de un uso excesivo en los pacientes con ERGE.

Es importante mencionar que hay un sesgo potencial por ser un estudio distribuido por redes sociales, donde posiblemente contestaron las personas con mayor acceso a estas o que tienen más síntomas; sin embargo, el título de la encuesta y los métodos de aplicación buscaron reducir dichos sesgos al mínimo. Esto abre las puertas a la realización de nuevos estudios que consideren otras variables y que busquen investigar poblaciones con menor acceso a redes sociales. También estamos conscientes de que la variación en el número de pacientes que contestaron por cada región del país puede presentar un sesgo de selección, sin embargo creemos que con el número de muestra logramos compensarlo en lo más posible. Este estudio se enfocó en el número de endoscopias realizadas; las indicaciones, los síntomas asociados o refractarios y los costos del uso de la endoscopia formarán parte de otros estudios posteriores.

Nuestro estudio tiene múltiples puntos a favor: es el primer estudio prospectivo en población general adulta mexicana que reporta el uso de la endoscopia por síntomas de reflujo gastroesofágico. Se empleó una muestra amplia que incluyó individuos de todas las zonas del país, de ambos sexos y con edades desde los 18 hasta los 72 años, reportando endoscopias/paciente/año. Nuestro estudio muestra que la endoscopia es el método más empleado en pacientes con síntomas de reflujo gastroesofágico en la población general de México. El $37.70 \%$ se ha realizado por lo menos una endoscopia por síntomas de reflujo en los últimos cinco años y esta representa el $52.4 \%$ de todos los estudios utilizados en la ERGE.

En conclusión, el uso de la endoscopia como método diagnóstico inicial en pacientes con síntomas de reflujo en México parece ser menor a lo reportado en la literatura internacional, aunque el número total de procedimientos por paciente es significativamente mayor. 


\section{Financiamiento}

La presente investigación no ha recibido ayudas específicas provenientes de agencias del sector público, sector comercial o entidades sin ánimo de lucro.

\section{Conflicto de intereses}

Los autores declaran que no hay conflicto de intereses.

\section{Responsabilidades éticas}

Protección de personas y animales. Los autores declaran que para esta investigación no se han realizado experimentos en seres humanos ni en animales.

Confidencialidad de los datos. Los autores declaran que han seguido los protocolos de su centro de trabajo sobre la publicación de datos de pacientes.

Derecho a la privacidad y consentimiento informado. Los autores declaran que en este artículo no aparecen datos de pacientes.

\section{Bibliografía}

1. Huerta-Iga F, Tamayo-de la Cuesta JL, Noble-Lugo A, Remes-Troche JM, Valdovinos-Díaz MA, Carmona-Sánchez RI; Grupo Mexicano para el Estudio de la Enfermedad por Reflujo Gastroesofágico 2011. Consenso mexicano de enfermedad por reflujo gastroesofágico (Parte I). Rev Gastroenterol Méx. 2012;77:193-213.
2. Huerta-Iga F, Bielsa-Fernández MV, Remes-Troche JM, Valdovinos-Díaz MA Tamayo-de la Cuesta JL; en representación del Grupo para el estudio de la ERGE 2015. Diagnóstico y tratamiento de la enfermedad por reflujo gastroesofágico: recomendaciones de la Asociación Mexicana de Gastroenterología. Rev Gastroenterol Méx. 2016;81(4):208-22.

3. Nabi Z, Reddy N. Update on endoscopic approaches for the management of gastroesophageal reflux disease. Gastroenterol Hepatol. 2019;15:369-76.

4. Valdovinos-Andraca F, Elizondo Rivera J, de la Torre-Bravo A, Abdo Francis JM. Segundo consenso mexicano para la enseñanza de la endoscopia gastrointestinal. Endoscopia. 2015;27(1):14-24.

5. Zhang M, Pandolfino JE, Zhou X, Tan N, Li Y, Chen M, et al. Assessing different diagnostic tests for gastroesophageal reflux disease: a systematic review and network metaanalysis. Ther Adv Gastroenterol. 2019;12:1-17.

6. Alcedo J. Concepto de enfermedad por Reflujo Gastroesofágico y Clasificación. En: Alcedo J, Mearin F, Balboa A, Mínguez M, Ciriza C, Montoro $\mathrm{M}$, et al. Documento de actualización de la Guía de Práctica Clínica sobre la Enfermedad por Reflujo Gastroesofágico en el adulto. Barcelona, España: Asociación Española de Gastroenterología; 2019. pp. 11-14.

7. Vakil N, van Zanten SV, Kahrilas P, Dent J, Jones R; Global Consensus Group. The Montreal definition and classification of gastroesophageal reflux disease: A global evidence-based consensus. Am J Gastroenterol. 2006;101:1900-20.

8. DeVault KP, Castell DO; American College of Gastroenterology. Updated guidelines for the diagnosis and treatment of gastroesophageal reflux disease. Am J Gastroenterol. 2005;100:190-200.

9. Mexico Endoscopy Market Statistics By Usability (Single-use/Disposable, Reusable), By Product (Endoscopes [Flexible Endoscopes, Rigid Endoscopes, Capsule Endoscopes], Visualization Systems, Endoscopic Ultrasound, Insufflator), By Application (Arthroscopy, Laparoscopy, GI Endoscopy, Obstetrics/Gynecology Endoscopy, ENT Endoscopy, Pulmonary Endoscopy), Industry Size, Share, Growth Trends \& Forecast, 2018-2024 [Internet]. Graphical Research Report; 2019 [citado el 30 de junio de 2020]. Disponible en: https://www.graphicalresearch.com/industry-insights/1149/mexico-endoscopy-market

10. Shaheen NJ, Weinberg DS, Denberg TD, Chou R, Qaseem A, Shekelle P; Clinical Guidelines Committee of the American College of Physicians Upper endoscopy for gastroesophageal reflux disease: Best practice advice from the clinical guidelines of the American College of Physicians. Ann Intern Med. 2012;157:803-16.

11. Schlottmann F, Herbella FA, Allaix ME, Rebecchi F, Patti MG. Treatment of gastroesophageal reflux disease. World J Surg. 2017:41(7):1685-90.

12. Ljungdalh JS, Rubin KH, Durup J, Houlind KC. Treatment of newly-diagnosed gastroesophageal reflux disease: a nationwide register-based cohort study. Scand J Gastroenterol. 2019;54(7):830-7.

13. Bardhan KD, Stanghellini V, Armstrong D, Berghöfer P, Gatz G, Mönnikes $H$. International validation of ReQuest in patients with endoscopy-negative gastroesophageal reflux disease. Digestion. 2007;75(Suppl 1):48-54. 